Communication

\title{
Comparative Atomic-Level Analysis of Sorption and Diffusion Properties of Membrane Materials on the Base of Polymer and Its Prepolymer: A Case Study of Polyheteroarylenes
}

\author{
Andrey V. Petrov (1) and Alexander M. Toikka *(i)
}

check for

updates

Citation: Petrov, A.V.; Toikka, A.M. Comparative Atomic-Level Analysis of Sorption and Diffusion Properties of Membrane Materials on the Base of Polymer and Its Prepolymer: A Case Study of Polyheteroarylenes. Membranes 2021, 11, 925. https:// doi.org/10.3390/membranes11120925

Academic Editor: Klaus Rätzke

Received: 18 October 2021

Accepted: 23 November 2021

Published: 25 November 2021

Publisher's Note: MDPI stays neutral with regard to jurisdictional claims in published maps and institutional affiliations.

Copyright: (c) 2021 by the authors. Licensee MDPI, Basel, Switzerland. This article is an open access article distributed under the terms and conditions of the Creative Commons Attribution (CC BY) license (https:/ / creativecommons.org/licenses/by/ $4.0 /)$.
Department of Chemical Thermodynamics and Kinetics, Institute of Chemistry, St. Petersburg State University, 199034 St. Petersburg, Russia; a.petrov@spbu.ru

* Correspondence: a.toikka@spbu.ru; Tel.: +7-812-428-4052

\begin{abstract}
The sorption properties of polymers and the mobility of penetrants are the main factors which determine the trans-membrane processes. Other factors concern the membrane material structure and chemical nature. In this paper, we consider the case of polymers with similar structure units, namely a polymer and its pre-polymer (polybenzoxazinoneimide and imide-containing polyamic acid). The available experimental data show a great difference in the pervaporation process using these two polymeric membranes. Some explanation of this difference can be found at the atomic-level study. A comparative analysis of the diffusion of water and isopropanol molecules was carried out using the density functional theory and molecular dynamics simulations.
\end{abstract}

Keywords: membranes; density functional theory; molecular dynamic simulation; polymers; prepolymers; sorption; mobility

\section{Introduction}

For the development of membrane technology, further investigation into new membrane materials is needed. In the case of polymeric membranes, which are used in pervaporation and related processes such as gas and vapor separation, new materials can be obtained in a few ways, e.g., changing the polymer matrix by embedding modifiers or creating composite materials. Other methods include the use of new polymers or the transformation of a polymer structure [1]. In recent decades, the heat treatment of polymers has been considered an effective method for their modification and application in membrane technology, see, e.g., [2-4]. In such processes the complex of separation, physical-chemical mechanical, and other properties of polymers may sufficiently differ from the properties of a pre-polymer. Such differences between properties of materials that have similar structural unites in polymer chains may be unexpected and requires additional analysis. In this paper, we consider this change of properties with the example of two membrane polymers, polybenzoxazinoneimide (PBOI) and its prepolymer, imidecontaining polyamic acid (PI-PAA). The objects of investigation are the sorption centers and mobility of solvents (penetrants, water, and isopropyl alcohols) which were analyzed using the quantum chemistry approach (density functional theory, DFT) and molecular dynamic simulation (MD).

\section{Materials and Methods}

It was shown in [5] that for the membranes on the base of polybenzoxazinone imide (PBOI) and its hydrolytically stable prepolymer, imide-containing polyamic acid (PI-PAA), there are significant differences in the pervaporation separation of water-isopropyl alcohol mixture. These membrane materials belong to the class of polymers of heteroaromatic structure or polyheteroarylenes, which are widely used in membrane technology due to their high thermal, mechanical, and chemical stability, combined with improved separation 
performance [6]. Another attractive feature of polyheteroarylenes is the possibility of a significant change in performance characteristics due to the modification of their structure. The separation efficiency is largely determined by repeating monomeric units, which may contain aromatic cycles (benzene, naphthalene) or various types of heterocycles, such as imides, imidazoles, oxazoles, etc. (see, e.g., recent works [4,6-9]). For the synthesis of new structures, methods of step-by-step synthesis are used, during which intermediate products (prepolymers) are formed, which also have certain membrane properties. In the case of polybenzoxazinones, such prepolymeres are polyamic acids that are also of interest for membrane technology. The synthesis of these compounds and the preparation of membranes on the base of imide-containing polyamic acid and polybenzoxazinoneimide were described in papers $[3,5,10,11]$. First of all, the conversion of PI-PAA into PBOI results in a significant rise in the separation factor, but a decrease in the trans-membrane fluxes [5].

In this work, using the methods of density functional theory (DFT) and classical molecular dynamics (MD), we quantitatively estimated the peculiarities of sorption centers in PBOI and PI-PAA and the mobility of water and isopropyl alcohol molecules.

Polymer dimers PBOI and PI-PAA were chosen as models to represent the polymer chains. This choice was determined by the fact that monomers in simulations are usually terminated by hydrogen atoms connected with head and tail atoms formed polymer chains. This may introduce significant distortions in the distribution of electron density in the polymer molecule. The influence of hydrogen atoms through 2-3 atoms of the main polymer chain have no significant influence on the next atoms. Therefore, we chose for the analysis an area connecting two monomers, in which all potential sorption centers for both polymers are presented. These areas of polymer chains are shown in Figures 1 and 2.

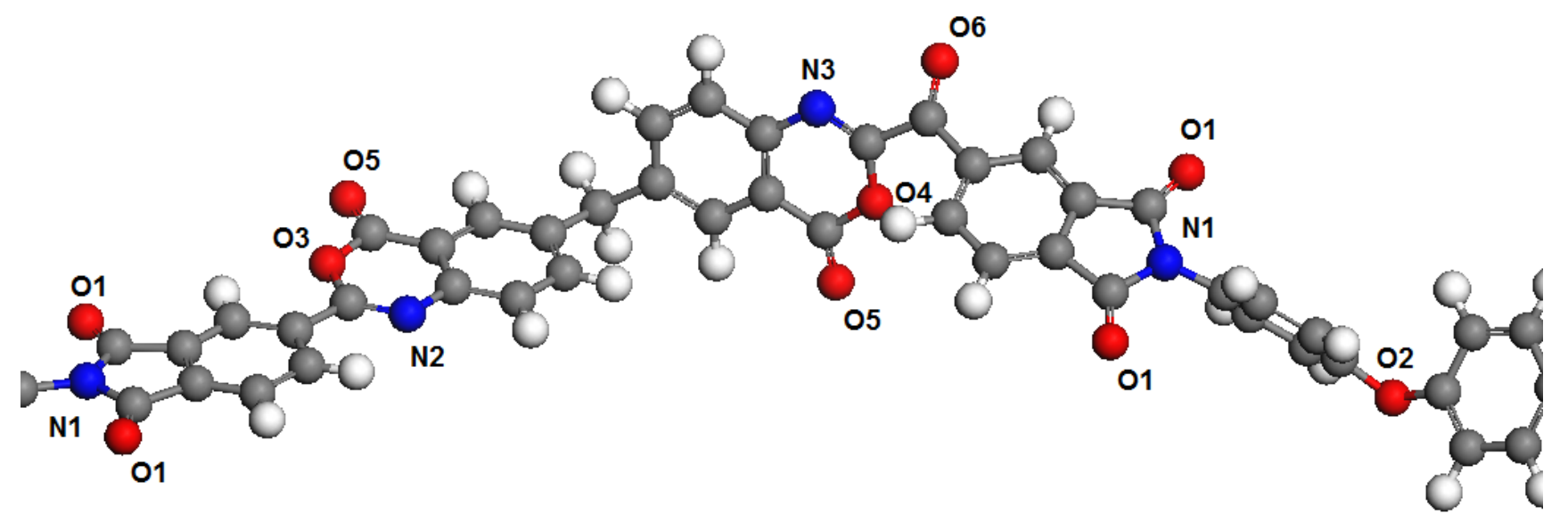

Figure 1. Fragment of the polymer chain PBOI with marked atoms according to Table 1.

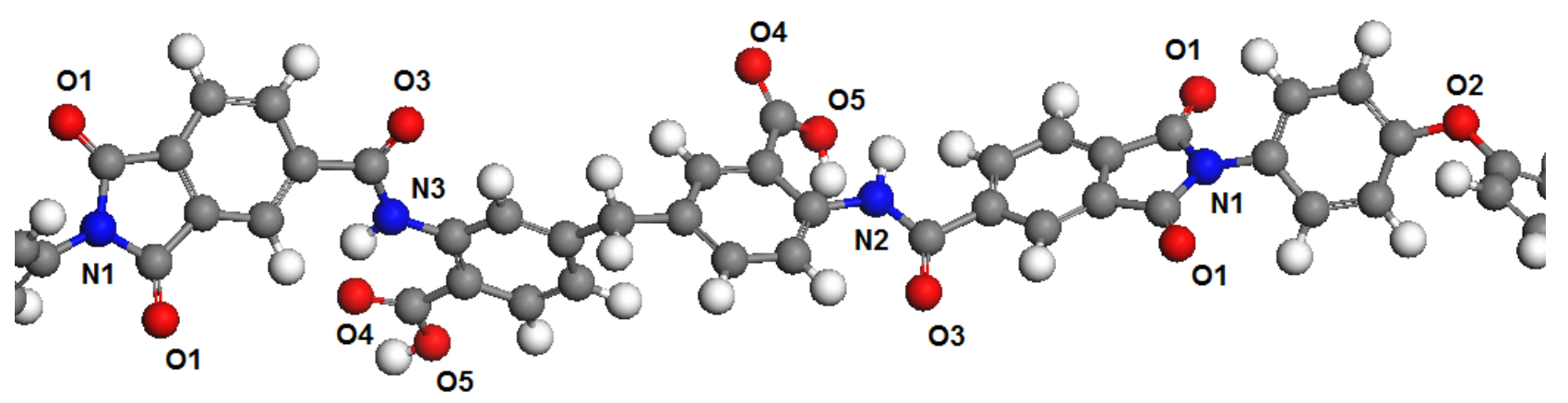

Figure 2. Fragment of the PI-PAA polymer chain with marked atoms according to Table 2. 


\section{Results}

At the first step, the electronic structure of dimers was calculated by the DFT method with full geometry optimization in water and isopropyl alcohol according to the COSMO model, where solvent is considered as a continuum with a definite permittivity. For the calculations, the $\mathrm{DMol}^{3}$ software module from the Materials Studio software package was used. The full-electron atomic basis DNP (4.4) and the PBE functional were used. Based on calculations of the electron density, the charge states of oxygen and nitrogen atoms were determined according to the Mulliken scheme. The values of charge states are presented in Tables 1 and 2.

Table 1. Charge states of sorption centers in PBOI (in charges of electron, e).

\begin{tabular}{ccccccccccc}
\hline Atom Name/Solvent & $\mathbf{N 1}$ & $\mathbf{N 2}$ & $\mathbf{N 3}$ & $\mathbf{O 1}$ & $\mathbf{O} 2$ & O3 & O4 & O5 & O6 \\
\hline Water & -0.289 & -0.267 & -0.234 & -0.444 & -0.446 & -0.447 & -0.433 & -0.444 & -0.397 \\
\hline Isopropanol & -0.291 & -0.265 & -0.231 & -0.439 & -0.452 & -0.448 & -0.427 & -0.436 & -0.390 \\
\hline
\end{tabular}

Table 2. Charge states of sorption centers in PI-PAA (in charges of electron, e).

\begin{tabular}{cccccccccc}
\hline Atom Name/Solvent & $\mathbf{N 1}$ & $\mathbf{N 2}$ & $\mathbf{N 3}$ & $\mathbf{O 1}$ & $\mathbf{O 2}$ & O3 & O4 & O5 \\
\hline Water & -0.292 & -0.299 & -0.288 & -0.443 & -0.440 & -0.499 & -0.513 & -0.424 \\
\hline Isopropanol & -0.294 & -0.311 & -0.290 & -0.439 & -0.454 & -0.493 & -0.439 & -0.443 \\
\hline
\end{tabular}

According to the results of calculations of the electronic structure in water, the oxygen atoms of the carboxyl groups (O4) and atoms connected by the carbonyl bond (O3) have charges of about $0.5 \mathrm{e}$ in PI-PAA. Such relatively high charges of oxygen atoms according to the same calculation are absent in the PBOI. The values of the charges of oxygen atoms (O1) in the groups presented in both polymers coincide both in water and in isopropanol. The values of the charges of the "bridging" oxygen atoms $(\mathrm{O} 2)$ are also close for both polymers in two media. Therefore, the contribution of these types of atoms to sorption practically does not determine the difference in sorption. Nitrogen atoms in the "pyrrole" positions (N1), which are presented in two polymer molecules, also have similar charges. Nitrogen atoms in the N2 and N3 position in PBOI have the lowest negative charges among all types of nitrogen atoms. The nitrogen atoms in PI-PAA also have increased negative charges compared to the nitrogen atoms in PBOI. The difference in the charge states of nitrogen atoms is apparently due to the close location to the oxygen atoms in the PBOI, which attract the electron density from the carbon atoms and, as a result, prevent the transfer of electron density to nitrogen atoms.

\section{Discussion}

Thus, determined charge states of dimers, as well as of water and isopropanol molecules, calculated according to the same calculation scheme, used as the basis for constructing a force field for calculations by the method of classical molecular dynamics. For this purpose, computational cells were constructed containing PBOI and PI-PAA dimers together with water and isopropanol molecules introduced into the system. The concentration of fluids in the polymers was about $1 \mathrm{wt} . \%$ : 25 dimer molecules, 25 water molecules, or 7 isopropanol molecules. To take into account short-range forces, the UFF force field [12] was used. The time of calculations was $5 \mathrm{~ns}$ with an integration step of $1 \mathrm{fs}$ at $298 \mathrm{~K}$ in the NVT ensemble. The computational cell for system PBOI-water is shown in Figure 3. 


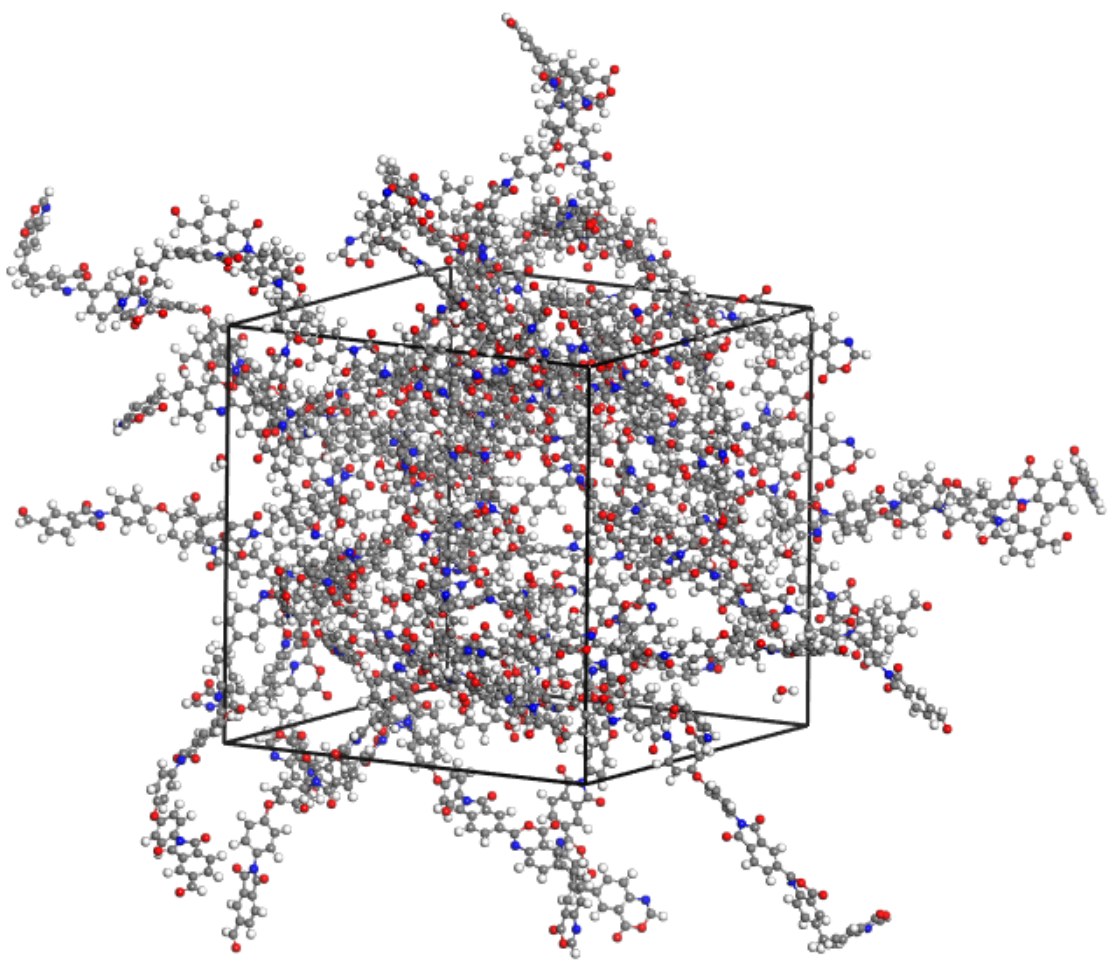

Figure 3. Cell for the system PBOI-water used for MD simulation.

Calculations of the mobility of penetrant molecules were estimated by the values of the root-mean-square displacements (MSD) Figures 4-7 presented in Table 3.

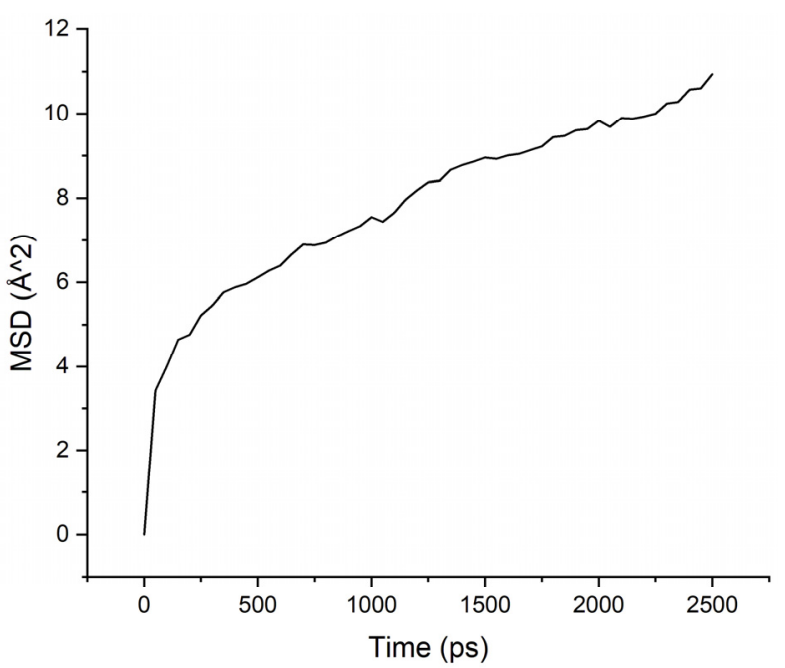

Figure 4. MSD for PAA-water system. 


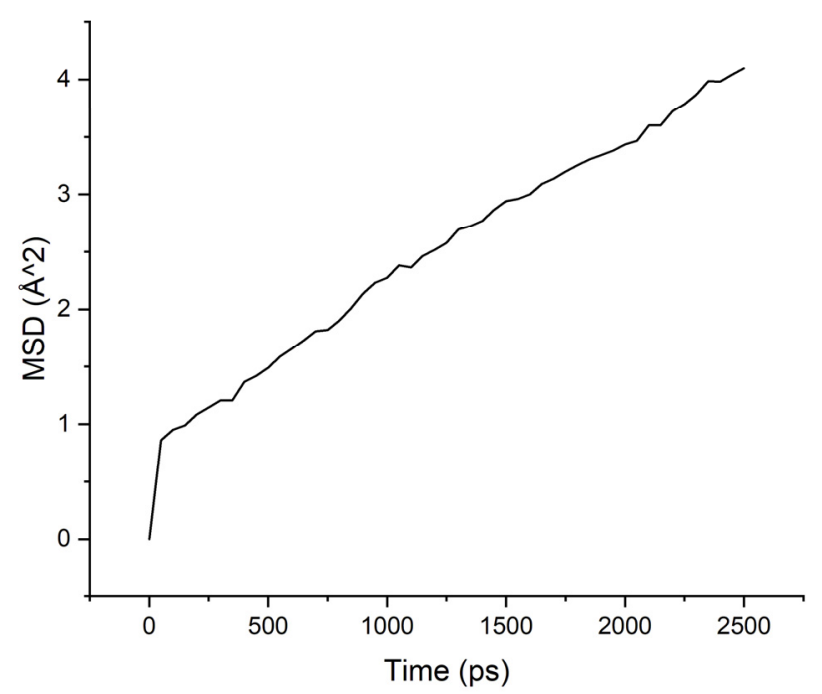

Figure 5. MSD for PAA-isopropanol system.

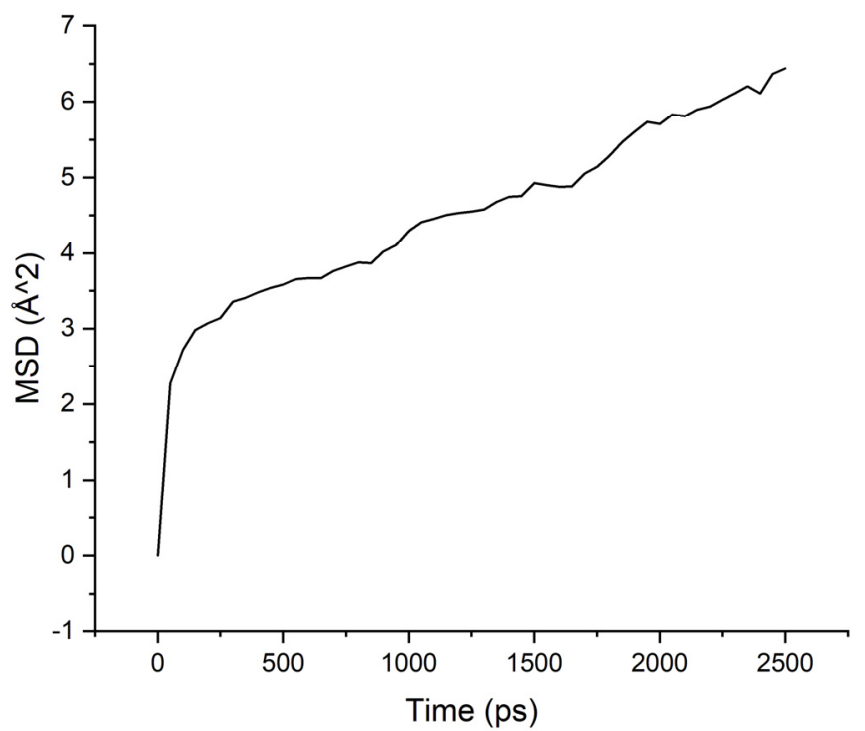

Figure 6. MSD for PBOI-water system.

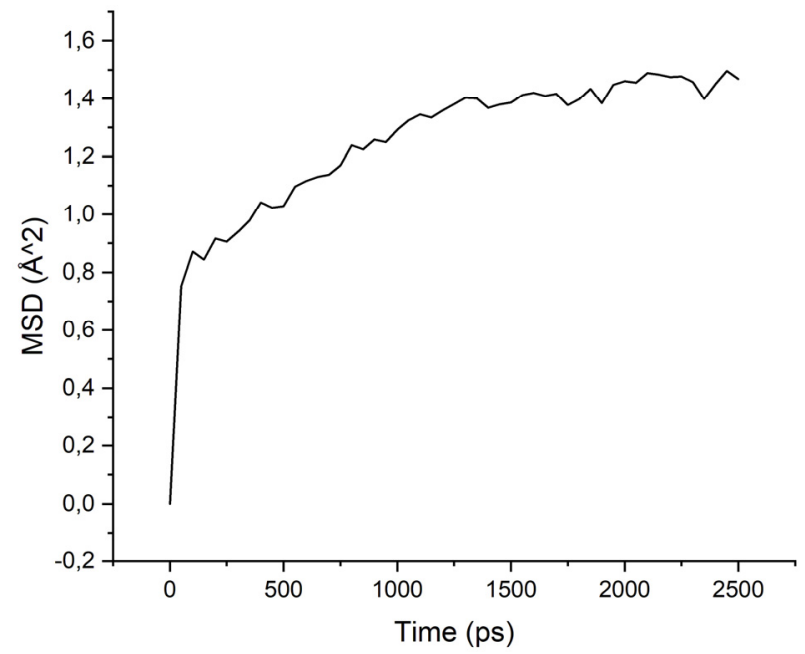

Figure 7. MSD for PBOI-isopropanol system. 
Table 3. Diffusion coefficients of water and isopropanol, calculated in PI-PAA and PBOI $\left(10^{-4} \AA^{2} / \mathrm{ps}\right)$.

\begin{tabular}{ccc}
\hline Polymer/Solvent & PI-PAA & PBOI \\
\hline Water & 4.595 & 2.633 \\
\hline Isopropanol & 2.260 & 0.397 \\
\hline
\end{tabular}

The MSD values demonstrate low mobility for penetrant molecules in a polymer environment. After an equilibration time of about $250 \mathrm{ps}$, the movements of molecules became stable and similar to oscillations near the sorption centers.

As can be seen from the results of calculations, the diffusion coefficients correspond to the experimental values: water molecules move faster than isopropanol molecules, both in PI-PAA and in PBOI. On the other hand, the behavior of isopropanol molecules in PBOI turned out to be unusual. A sharp decrease in mobility, almost to a complete stop (only vibrations were observed), shows that PBOI is a very effective material for the sorption of isopropanol. At the same time, the difference in the dipole moments of water and isopropanol molecules $(1.84 \mathrm{D}$ and $1.66 \mathrm{D}$, respectively) also corresponds to a significant difference in the sizes of these molecules. The data of radial distribution functions (RDF) between isopropanol (center of mass) and sorption centers in PBOI presented in Table 4. The distances were snapshotted after $0.5,1.5$ and $2.5 \mathrm{~ns}$ of simulation time.

Table 4. Maximums of radial distribution functions between isopropanol and sorption centers in PBOI $(\AA)$.

\begin{tabular}{cccccccccc}
\hline Soprtion Center & N1 & N2 & N3 & O1 & O2 & O3 & O4 & O5 & O6 \\
\hline RDF after 0.5 ns & 3.97 & 4.29 & 4.71 & 3.35 & 3.43 & 5.13 & 3.79 & 3.79 & 4.17 \\
\hline RDF after 1.5 ns & 4.09 & 3.89 & 4.07 & 3.49 & 3.83 & 3.69 & 3.71 & 3.71 & 3.53 \\
\hline RDF after 2.5 ns & 4.47 & 3.89 & 3.91 & 3.83 & 3.49 & 4.85 & 3.65 & 3.65 & 3.85 \\
\hline
\end{tabular}

The oxygen atoms of PBOI play the most attractive role for isopropanol molecules because of their increased negative charges and open locations on the sides of the polymer chain. Nitrogen atoms (N1) are included in the chain and have space difficulties for interaction with big molecule of isopropanol. Nevertheless, atoms N2 and N3 have relatively small negative charge, but very good location on the side of polymer chain and can interact with fluid. The atom $\mathrm{O} 5$ and atoms $\mathrm{O} 3 \mathrm{O} 4$, when close to an atom with great negative charges, significantly attract the isopropanol molecule. The most negative charged atom $\mathrm{O} 2$, with an electron density collected from the nearest benzoic circles, has the most close contact with isopropanol molecules.

As a result, the complex of these factors leads to an increase in the separation properties of membranes on the base of PBOI. The decrease in the flux for this membrane could be connected with the higher density of PBOI. All these conclusions are in a good agreement with the experimental data of the work [5]. Thus, we have shown that differences in the membrane characteristics of polymers with common structural units, in this case, a polymer and prepolymer, can be predicted at an atomic level using calculations based on DFT and molecular dynamics simulations.

\section{Conclusions}

In this paper, we presented some results that may explain the significant differences in the membrane properties of two polymers with similar structure units in a polymer chain: polybenzoxazinoneimide (PBOI) and its pre-polymer imide-containing polyamic acid (PI-PAA). The experimental data on pervaporation water-isopropyl alcohol systemusing these two polymeric membranes were recently published in paper [5]. The study on the base of density functional theory and molecular dynamic simulation reflects a sharp increase in sorption properties during the transition from PI-PAA to a PBOI. The electronic 
structure was calculated by the DFT method with full optimization of the geometry in water and isopropyl alcohol according to the COSMO model. Molecular dynamic simulation was performed for cells containing PBOI and PI-PAA dimers, water, and isopropanol molecules.

In particular, the results show that the mobility of alcohol molecules, unlike water, in PBOI, is very low, which determines the increase in the selectivity of this membrane material during the dehydration of isopropanol. In general, the results of the comparative atomic-level analysis of sorption and diffusion properties are in agreement with experimental data on pervaporation [5]. Accordingly, such study may be useful for a priory qualitative analysis of some structural and dynamic factors influencing penetrant behavior in pervaporation.

Author Contributions: Performed calculations and discussed the results, A.V.P. and A.M.T.; Analyzed and presented the data, A.V.P.; Supervised the work and discussed the results, A.M.T.; All authors contributed to the preparation of the text of the paper. Data curation, A.V.P.; Formal analysis, A.V.P.; Investigation, A.V.P. and A.M.T.; Methodology, A.V.P.; Supervision, A.M.T.; Validation, A.M.T.; Writing — original draft, A.V.P. and A.M.T.; Writing—review and editing, A.V.P. and A.M.T. All authors have read and agreed to the published version of the manuscript.

Funding: This work was funded Russian Foundation for Basic Research (RFBR grant 19-03-00375) for the support of this study.

Institutional Review Board Statement: Not applicable.

Informed Consent Statement: Not applicable.

Data Availability Statement: All necessary data are contained in the paper.

Acknowledgments: Research was carried out using computational resources provided by Resource Center "Computer Center of SPbU".

Conflicts of Interest: The authors declare no conflict of interest.

\section{References}

1. Baker, R.W. Membrane Technology and Applications, 3rd ed.; Wiley: Chichester, UK, 2012.

2. Ohya, H.; Kudryavtsev, V.V.; Semenova, S.I. Polyimide Membranes; Gordon \& Breach Publishers: New York, NY, USA, 1996.

3. Goikhman, M.; Gofman, I.; Tikhonova, L.; Mikhailova, M.; Kudryavtsev, V.; Laius, L.A. Synthesis and Properties of Poly(Benzoxazinoneimide)s. Polym. Sci. Ser. A 1997, 39, 197-202.

4. Yang, S.-Y. (Ed.) Advanced Polyimide Materials: Synthesis, Characterization and Applications; Elsevier: Amsterdam, The Netherlands, 2018.

5. Pulyalina, A.Y.; Polotskaya, G.A.; Rostovtseva, V.A.; Goikhman, M.Y.; Podeshvo, I.V.; Shugurov, S.M.; Zubakina, E.A.; Toikka, A.M. Sorption Properties and Transport Parameters of Membranes Based on Polybenzoxazinoneimide and Its Prepolymer. Pet. Chem. 2017, 57, 318-326. [CrossRef]

6. Pulyalina, A.Y.; Polotskaya, G.A.; Toikka, A.M. Membrane materials based on polyheteroarylenes and their application for pervaporation. Russ. Chem. Rev. 2016, 85, 81-98. [CrossRef]

7. Álvarez, C.; Lozano, Á.E.; Juan-y-Seva, M.; de la Campa, J.G. Gas separation properties of aromatic polyimides with bulky groups. Comparison of experimental and simulated results. J. Membr. Sci. 2020, 602, 117959. [CrossRef]

8. Xu, S.; Wang, Y. A prospective study on thermally-cyclodehydrated poly(imide-oxadiazole) membranes for pervaporation dehydration. Membr. Sci. 2018, 549, 184-191. [CrossRef]

9. Zhang, C.; Li, P.; Cao, B. Effects of the side groups of the spirobichroman-based diamines on the chain packing and gas separation properties of the polyimides. J. Membr. Sci. 2017, 530, 176-184. [CrossRef]

10. Polotskaya, G.A.; Kuznetsov, Y.P.; Goikhman, M.Y.; Podeshvo, I.V.; Maricheva, T.A.; Kudrjavtsev, V.V. Pervaporation membranes based on imide-containing poly(amic acid) and poly(phenylene oxide). J. Appl. Polym Sci. 2003, 89, 2361-2368. [CrossRef]

11. Polotskaya, G.A.; Goikhman, M.Y.; Podeshvo, I.V.; Kudryavtsev, V.V.; Pientka, Z.; Brozova, L.; Bleha, M. Gas transport properties of polybenzoxazinoneimides and their prepolymers. Polymer 2005, 46, 3730-3736. [CrossRef]

12. Rappe, A.K.; Casewit, C.J.; Colwell, K.S.; Goddard III, W.A.; Skiff, W.M. UFF, a full periodic table force field for molecular mechanics and molecular dynamics simulations. J. Am. Chem. Soc. 1992, 114, 10024-10035. [CrossRef] 